\title{
A Stability-oriented Design Method for Virtual Impedance Loop in Droop-controlled Converters
}

\author{
Alberto Bolzoni, Roberto Perini \\ Energy Department \\ Politecnico di Milano \\ via La Masa 34, 20156 Milano, Italy \\ alberto.bolzoni@polimi.it,roberto.perini@polimi.it
}

\author{
Luisa Frosio \\ EPS Elvi Energy - Electro Power System Group \\ Milano, Italy \\ luisa.frosio@eps-mail.com
}

\begin{abstract}
In this paper, a novel strategy for virtual impedance design in droop-connected converters is analysed; the method is based on the stability analysis of equivalent system loops and aims at defining feasible operating regions of the converter depending on the control parameters. The effect of the tuning on the regulating performances of the system then has been tested in a simulation environment.
\end{abstract}

Keywords - Microgrids, converter control, virtual impedance, stability assessment.

\section{INTRODUCTION}

Grid-connected converters are becoming significant components in modern electrical power systems, thanks to the operational flexibility they can provide and the possibility to interface renewables or storage units. A higher penetration of sustainable resources in electrical grids will foster the evolution of energy production towards a reduction in the carbon footprint.

One of the major research topics in the field of interface converters is represented by droop control. This type of regulation introduces a linear dependence between active power and frequency (as well as reactive-power and voltage amplitude); this has the following advantages:

- Island or grid-connected operations are allowed with no changes in the control structure.

- Equal power sharing between units during island operations

One of the major drawbacks of droop-connected converters is associated to the sensitivity of the control with respect to the output impedance of the system. Thus an additional control loop called virtual impedance was proposed in literature [1], in order to improve dynamical performances of the control and increase power decoupling, especially in low-voltage networks with a significant resistive short-circuit impedance. Different approaches can be found in literature to design this additional control loop [2] - [3]. In this paper a novel approach for virtual impedance sizing is proposed for a converter connected to an external low-voltage grid. The technique is based on the identification of a simplified transfer function that represents the whole system dynamical model; the reduction of some terms is carried out to build a manageable model for the virtual impedance parameter design, taking into account some constraints on the system stability.

The paper is organized as follows: in Part II the system is described and the dynamical model is derived. Part III deals with the design method based on closed-loop stability while in Part IV the model is validated through numerical simulations in Matlab - Simulink. Part V contains the conclusion of the work.

\section{SYSTEM DESCRIPTION}

Consider a three-phase converter connected to an external Micro-grid where primary voltage and frequency regulations are performed by means of droop equations (Fig. 1). According to the droop definition, each converter (defined by the index $i$ ) regulates the voltage $\bar{v}_{o}$ across the filter capacitor by changing the angular frequency $\omega$ and the voltage amplitude $V_{o}$ as a function of the injected active and reactive powers respectively.

$$
\begin{aligned}
& \omega_{i}=\omega_{i}^{*}-m_{i} \cdot\left(P_{i}-P_{i r e f}\right) \\
& V_{o i}=V_{o i}^{*}-n_{i} \cdot\left(Q_{i}-Q_{i r e f}\right)
\end{aligned}
$$

Thanks to the properties of the droop, the converter contributes to the grid regulation when it is operated in island mode, while it allows the control of active and reactive power injections through setpoints when connected to a higher power grid. Fig. 2 shows the complete control system of the inverter and the model of the physical connection circuit [4]. The design properties of the controllers and the physical values of the circuit elements are reported in Table I.

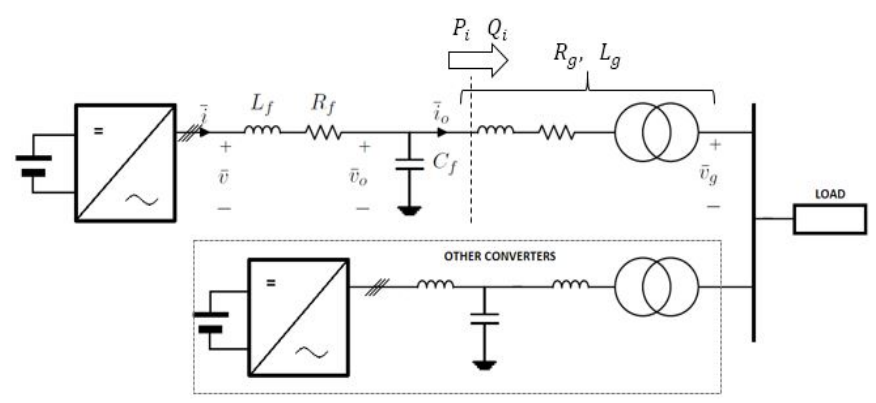

Fig. 1: Droop-interfaced converters connected to a Microgrid system 


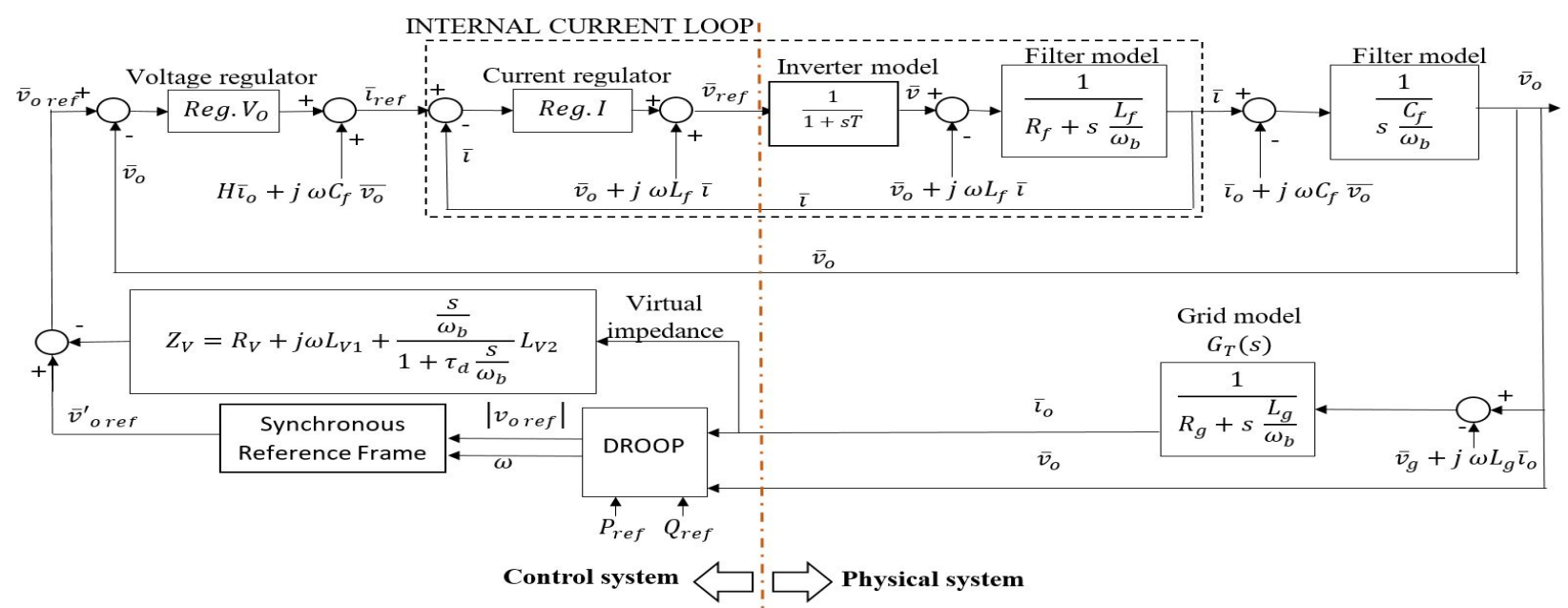

Fig. 2: Equivalent model of the control system. All the quantities refer to the circuit in Fig. 1. The constant $\mathrm{H}$ represents the non-ideal current compensation in the voltage loop

TABLE I. SYSTEM PARAMETERS

\begin{tabular}{c|c}
\hline \hline \multicolumn{2}{c}{ Reference values } \\
\hline \hline Rated $V b[\mathrm{~V}], A b[\mathrm{kVA}], \omega b[\mathrm{rad} / \mathrm{s}]$ & $200 ; 2.4 ; 2 \pi \cdot 50$ \\
\hline$R_{f}, L_{f}, C_{f}$ [p.u.] & $0.0073 ; 0.045 ; 0.052$ \\
\hline$R_{g}, L_{g}$ [p.u.] & $0.049 ; 0.024$ \\
\hline Current loop cut-off frequency $[\mathrm{rad} / \mathrm{s}]$ & $2 \pi \cdot 350$ \\
\hline Current loop phase margin $[\mathrm{deg}]$ & 90 \\
\hline Voltage loop cut-off frequency $[\mathrm{rad} / \mathrm{s}]$ & $2 \pi \cdot 35$ \\
\hline Voltage loop phase margin $[\mathrm{deg}]$ & 100 \\
\hline$\omega_{i}^{*}, V_{o i}^{*}$ [p.u.] & $1.0008 ; 1.001$ \\
\hline$m_{i}, n_{i}$ [p.u.] & $0.01 ; 0.0167$ \\
\hline
\end{tabular}

The system is described considering its state variables expressed as Park vectors in a $d q$ synchronous reference frame; $s$ represents the derivative operator in the Laplace domain. The system is composed by an internal current controller $\operatorname{Reg}_{I}$ which regulates current $\bar{l}$ through the filter inductor, while the controller $\operatorname{Reg}_{V}$ regulates the capacitor voltage $\bar{v}_{o}$; traditional PI structure is used for both controllers and feedforward compensation is implemented. Thus the controllers can be represented by:

$$
\begin{gathered}
\bar{l}_{\text {ref }}=\left(\bar{v}_{\text {oref }}-\bar{v}_{o}\right) \cdot \operatorname{Reg}_{V_{o}}+H \bar{l}_{o}+j \omega C_{f} \bar{v}_{o} \\
\bar{v}_{r e f}=\left(\bar{l}_{\text {ref }}-\bar{\imath}\right) \cdot \operatorname{Reg}_{I}+\bar{v}_{o}+j \omega L_{f} \bar{l}
\end{gathered}
$$

where $\operatorname{Reg}_{V_{o}}$ and $\operatorname{Reg}_{I}$ synthetically represent the transfer function of the voltage and current PI regulators. The model also takes into account the effect of non-ideal current compensation in the voltage loop, considered through the term $H<1$. As shown in literature [5], this term has a strong influence on the system stability and thus it needs to be taken into account in the design of the controllers.

The two external feedbacks are associated to the virtual impedance and the droop. The droop slowly changes the voltage reference $\bar{v}^{\prime}{ }_{\text {o ref }}$ as a function of the external system conditions according to (1) and (2), but its effectiveness strongly depends on the output impedance of the converter, which has to show a predominant inductive nature. Thus the additional loop of virtual impedance is introduced in order to adapt the equivalent impedance seen by the converter even during time-changing conditions of the grid. As the droop is generally characterized by a slower dynamic, its effect will not be included into the design model and the droop reference will be assumed constant both in amplitude and frequency.

Consider a virtual impedance with the following configuration:

$$
\bar{Z}_{V}=R_{V}+j \omega L_{V 1}+\frac{s / \omega_{b}}{1+\tau_{d} \cdot s / \omega_{b}} L_{V 2}
$$

The equivalent control structure of the virtual impedance loop can be expressed by:

- a proportional term (virtual resistance) defined by the coefficient $R_{V}$;

- a $d q$ coupling term (static virtual reactance), proportional to the coefficient $L_{V 1}$;

- an approximate derivative term (dynamic virtual reactance) proportional to the coefficient $L_{V 2}$.

Time constant $\tau_{d}$ can be introduced in order to limit the bandwidth of the dynamic virtual reactance and to have a feasible system; anyway, as its effect is perceivable only at a higher frequency with respect to the ones considered in the design phase, it will be neglected. So the following equation is considered:

$$
\bar{v}_{o r e f}=\bar{v}_{o r e f}^{\prime}-\left(R_{V}+j \omega L_{V 1}+\frac{s}{\omega_{b}} L_{V 2}\right) \cdot \bar{\iota}_{o}
$$

As regards the internal dynamics of the physical circuit, they can be described considering the following equations, which refer to the converter filter:

$$
\begin{gathered}
\bar{v}=\bar{v}_{o}+R_{f} \bar{\imath}+j \omega L_{f} \bar{l}+\frac{s}{\omega_{b}} L_{f} \bar{l} \\
\bar{\imath}=\bar{\iota}_{o}+j \omega C_{f} \bar{v}_{o}+\frac{s}{\omega_{b}} C_{f} \bar{v}_{o}
\end{gathered}
$$


As regards the model of the external grid, it can be represented by a three phase symmetrical set of voltages, expressed by the Park vector $\bar{v}_{g}$. The converter is supplying power to the grid through an impedance characterized by a resistive part $\left(R_{g}\right)$ and an inductive one $\left(L_{g}\right)$. Thus in the Park domain it is possible to determine the characteristic equation of the grid.

$$
\bar{v}_{o}=\left(R_{g}+L_{g} \frac{s}{\omega_{b}}+j \omega L_{g}\right) \cdot \bar{\iota}_{o}+\bar{v}_{g}
$$

All the equations are synthetically represented in the equivalent control scheme of Fig. 2, which has been used for the derivation of the simplified design model of the virtual impedance.

\section{CONTROL LOOPS APPROACH}

In this section, a method for virtual impedance design is developed. Differently from other techniques reported in literature, the model aims at analysing not only the effect of virtual impedance on circuit behavior in terms of power couplings, but also the stability properties of the voltage loop after the introduction of the additional virtual impedance feedback. Starting from the model of Fig. 2, it is possible to analytically derive the equivalent dynamical system for the direct and quadrature axis (Fig. 3); in this way, it is possible to highlight the effect of the different terms in the equivalent control loops of the system. The proposed design is composed of two different phases:

1. The virtual resistance and the dynamic virtual reactance are designed as to guarantee a sufficient phase margin and adequate cut-off frequency for the open-loop transfer function of the voltage control.

2. The virtual reactance design is carried out considering the decoupling between active and reactive power.

\section{A. First phase of the design process}

If we refer to the external voltage loop of Fig. 2, it is plain that both virtual resistance and dynamic virtual reactance change the stability properties of the system. A negative value of virtual resistance $R_{V}$ can introduce a higher decoupling effect as it compensates output resistive components, but it changes feedback properties.

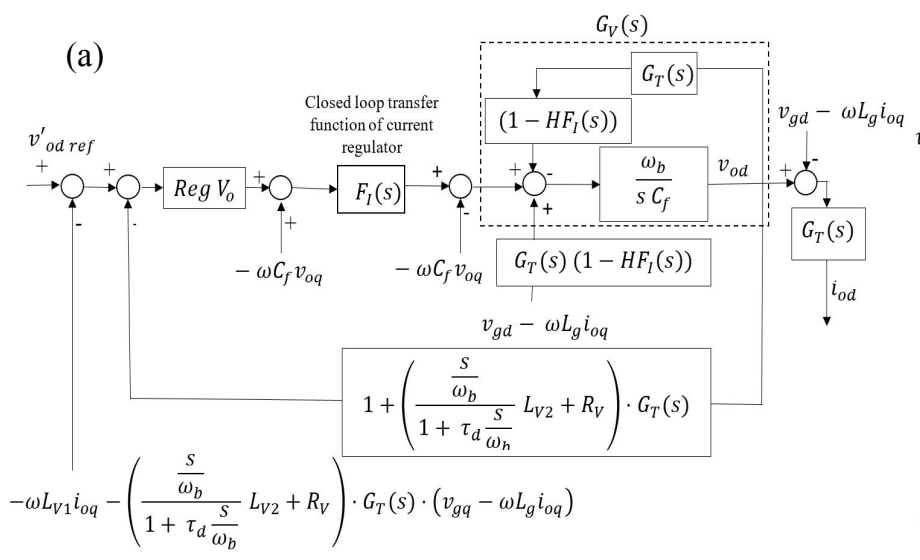

For design purposes the following additional hypotheses are considered:

- The dynamics of the internal current loop can be neglected, as its cut-off frequency is much higher than the considered frequency range.

- An ideal derivative is considered for the dynamic virtual reactance $\left(\tau_{d}=0\right)$, as its time constant is in general lower than the cut-off frequency of the voltage loop.

From Fig. 3 it is possible to derive the open-loop transfer function which includes the effects associated to virtual resistance and dynamic virtual reactance:

$$
\begin{aligned}
& O_{1}(s) \approx \operatorname{Reg}_{V o}(s) \cdot G_{V}(s) \cdot \\
& \quad\left(1+R_{V} G_{T}(s)+\frac{s}{\omega_{b}} L_{V 2} G_{T}(s)\right)
\end{aligned}
$$

where $G_{T}(s)$ represents the grid admittance in the Laplace domain (Fig. 2):

$$
G_{T}(s)=\frac{1}{\frac{s}{\omega_{b}} L_{g}+R_{g}}
$$

while $G_{V}(s)$ stands for the equivalent model of the filter capacitor when the non-ideal compensation of the current $\bar{l}_{o}$, represented by the term $H$, is included in the design of the voltage controller. This equation can be analytically derived considering the equivalent transfer function of the load that has to be regulated by the voltage controller, and it is modelled as an additional internal feedback in the voltage loop, as shown in Fig. 3.

$$
G_{V}(s)=\frac{\frac{\omega_{b}}{s C_{f}}}{1+\frac{\omega_{b}}{s C_{f}} G_{T}(s)(1-H)}
$$

By analysing the transfer function $O_{1}(s)$ it is possible to see that $L_{V 2}$ does not produce any effect at the equilibrium, while a negative value of virtual resistance $R_{V}$ improves the decoupling at steady state between active and reactive powers acting as a reduction factor of the voltage feedback. In particular when $s \cong$ 0 this factor equals:

(b)

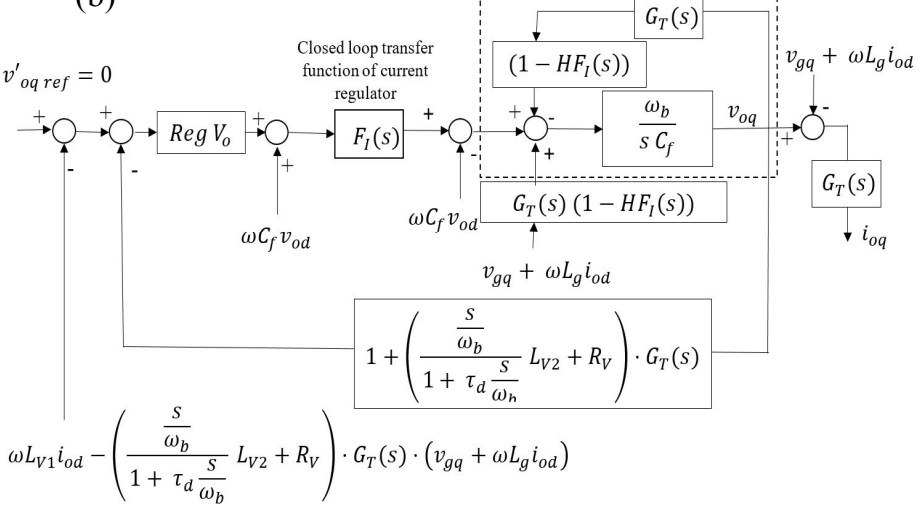

Fig. 3: Model elaboration for the analysis of the stability effects of virtual impedance on the converter dynamics, considered separately for the direct (a) and quadrature (b) components. $\mathrm{F}_{\mathrm{I}}(\mathrm{s})$ represents the closed loop transfer function of the current feedback reported in Fig. 2. 


$$
K_{V}=1+R_{V} G_{T}(0)=\left(1-\left|R_{V}\right| / R_{g}\right)
$$

Thus the following condition needs to be verified in order to have a negative feedback on the equivalent voltage loop:

$$
\left|R_{V}\right|<R_{g}
$$

Moreover a certain margin must be kept in order to take into account possible uncertainties associated to the values of the grid mpedance.

Besides the stability condition expressed by (14), the values for the design of the virtual resistance $R_{V}$ and dynamic virtual reactance $\frac{s}{\omega_{b}} L_{V 2}$ can be determined considering the phase margin and cut-off frequency of the voltage loop. The introduction of a negative virtual resistance $R_{V}$ produces an improvement of the loop phase margin only if it is combined to a sufficient dynamic virtual reactance, as shown in Fig. 4-a; if this condition is not met, the introduction of a negative $R_{V}$ may cause a reduction of the system phase margin (Fig. 4-a) and significant changes in the equivalent band-pass of the voltage loop, as in Fig. 4-(b). As regards $L_{V 2}$, its introduction improves the frequency behaviour of the voltage feedback as it can compensate the cut-off frequency reduction associated to virtual resistance.

This behaviour is mainly due the the presence of resonance phenomena for the filter at low frequency, as it is is shown in Fig. 5; this cause the high sensitivity of the voltage loop phase margin and cut-off frequency for with respect to parameters variations, as shown in Fig. 4-(a) and 4-(b).

The design criterion adopted for the virtual resistance $R_{V}$ may be associated to the minimum desired phase margin on the voltage loop; as for the parameter $L_{V 2}$ associated to the dynamic virtual reactance, its value can be chosen considering the desired cut-off frequency for the voltage loop, also considering the effect of the resonance amplification. Here it has been chosen a phase margin equal to 120 degrees and a cut-off frequency of $200 \mathrm{rad} / \mathrm{s}$. Thus the following values can be considered for the nominal design of the parameters: $R_{V n}=-0.025$ p.u. and $L_{V 2 n}=$ 0.04 p.u.

\section{B. Second phase of the design process}

As regards the static virtual reactance $j \omega L_{V 1}$, it does not produce any significant variation in the voltage feedback; anyway the introduction of this control parameter increases the coupling between direct and quadrature components into the system. This behavior can be derived considering the mutual effect of the direct and quadrature voltage control loops. In Fig. 6 it has been reported the equivalent model of the whole system which includes direct and quadrature axis; the model has been obtained by considering mutual coupling terms between Fig. 3a and Fig. 3-b. In order to simplify the design process some of the internal couplings in the voltage loop, associated to non-ideal current compensation, have been neglected as they are numerically negligible.

If we focus on the coupling feedback reported in Fig. 6, it is possible to see that the static virtual reactance $j \omega L_{V 1}$ acts as an
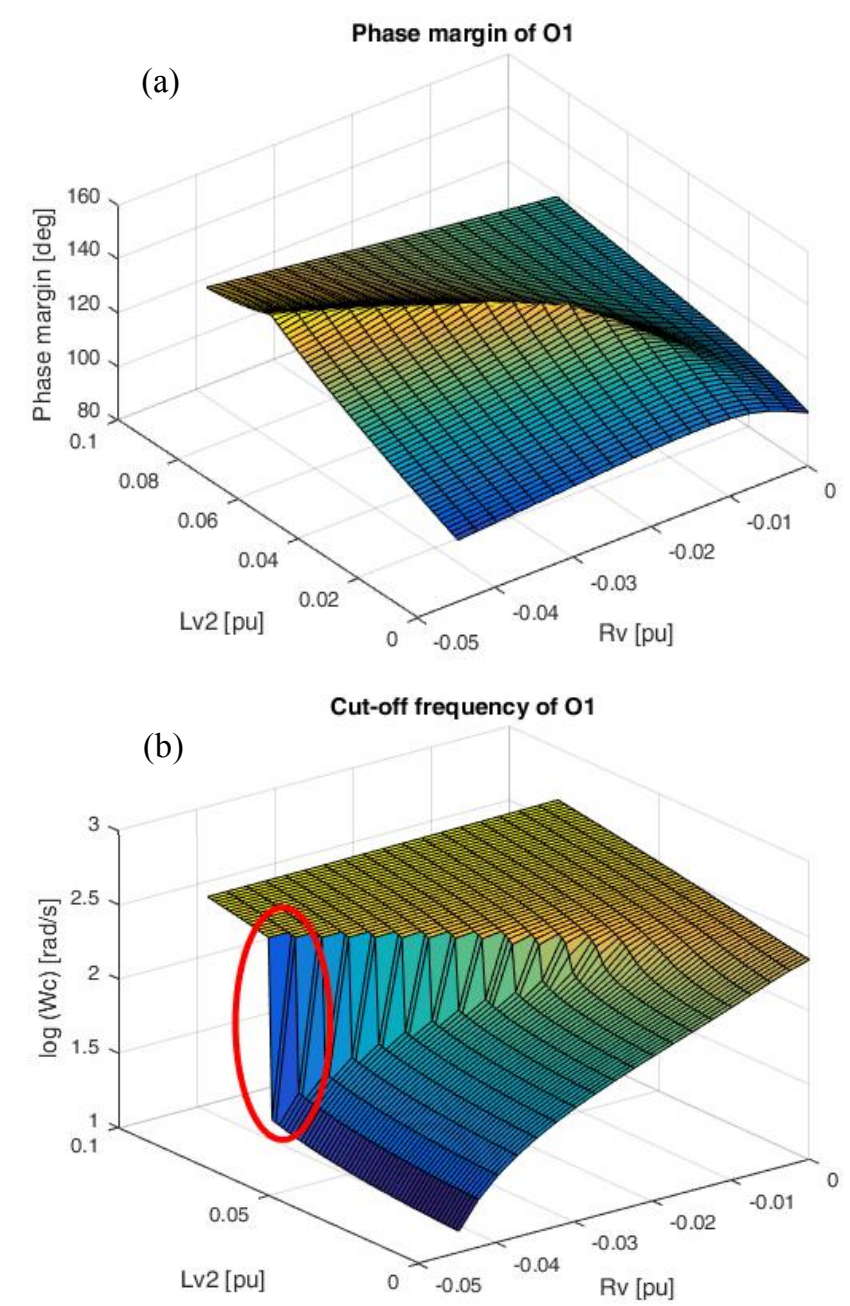

Fig. 4: Voltage loop phase margin (a) and cut-off frequency (b) as a function of the virtual resistance and dynamic virtual reactance coefficients in the stability region. The following ranges are considered:

$R_{V} \in[-0.047$ p.u. $; 0]=\left[-0.95 R_{g} ; 0\right]$

$L_{V 2} \in[0 ; 0.084$ p.u. $]=\left[0 ; 3.5 L_{g}\right]$

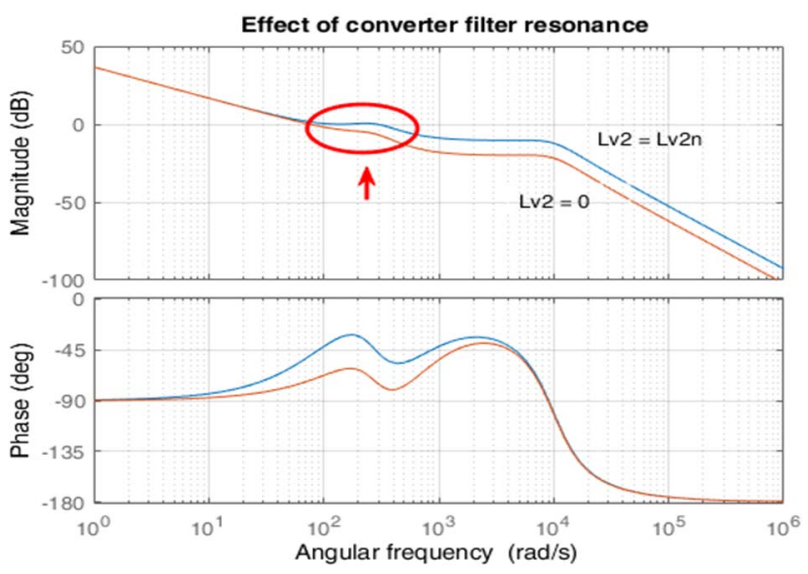

Fig. 5: Virtual impedance introduction may significantly change the cut-off frequency of the system due to the amplification of the effects associated to resonance phenomena on the converter filter.

The case shown refers to $R_{V}=R_{V n}$. 


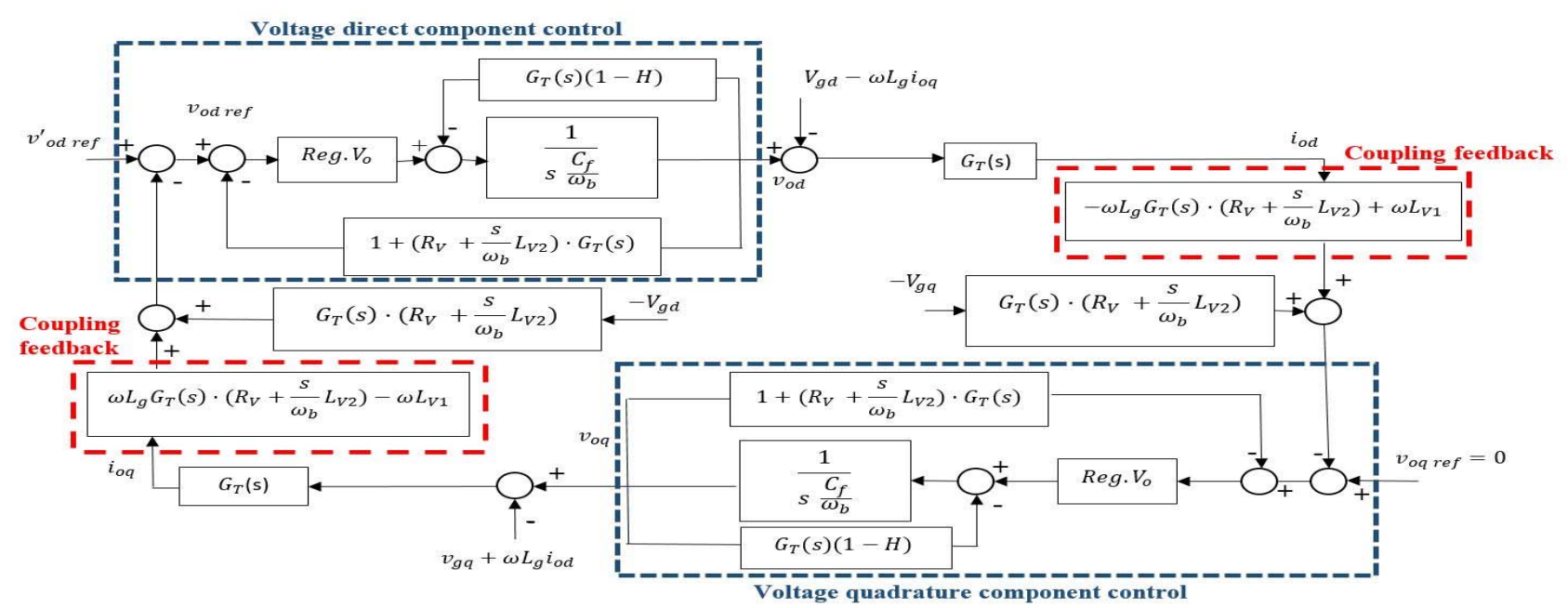

Fig. 6: Elaboration of the equivalent control system for the design of static virtual reactance term $j \omega \mathrm{L}_{\mathrm{V} 1}$. It is possible to see in the scheme the effect of the static virtual reactance, which increases the coupling between $\mathrm{i}_{\text {oq }}$ and $\mathrm{v}_{\text {od }}$; this makes the system more inductive.

additional coupling term between the quadrature current component $i_{o q}$ and the direct voltage component $v_{o d}$. Actually a more inductive equivalent output inductance results in a higher coupling between $i_{o q}$ and $v_{o d}$, according to basics electrotechnics; this results in a higher decoupling between active and reactive powers. If we consider the coupling $i_{o q}-v_{o d}$ at steady state $(s=0)$ with a negative virtual resistance $\left(R_{V}=\right.$ $\left.-\left|R_{V}\right|\right)$, it is equal to:

$$
k_{q}=-\omega L_{g} \frac{\left|R_{V}\right|}{R_{g}}-\omega L_{V 1}
$$

while the coupling between $i_{o d^{-}} v_{o d}$ at steady state can be expressed as the inverse of the transfer function $G_{T}(s=0)$.

$$
k_{d}=R_{g}
$$

Thus by defining the desired $\frac{\left|k_{q}\right|}{k_{d}}$, it is possible to choose the value of the nominal $L_{V 1 n}$; here it has been chosen $\frac{\left|k_{q}\right|}{k_{d}}=2$ and $L_{V 1 n}=0.08 \mathrm{pu}$. Thus it is easy to recognize that the combination of a negative value for virtual resistance and positive static virtual reactance results in a higher equivalent output reactance for the system, which produces a higher decoupling between active and reactive powers.

\section{MODEL VALIDATION}

In order to verify the effectiveness and correctness of the proposed design method, some simulations have been performed in the Matlab-Simulink environment to test the interactions of the virtual impedance parameters in the complete control system of the converter.

In Fig. 7 it has been studied the effect of the virtual resistance under subsequent step changes of the reference active power. At the beginning the transient has been simulated with no virtual impedance, as benchmark. By introducing static virtual reactance $j \omega L_{V 1}$ and a negative value of virtual resistance $R_{V}$, it is possible to observe a significant increase in the decoupling between the steady state values of active and reactive powers. This can be seen considering the reduction of the change in reactive power produced by active transients: as the virtual resistance becomes more negative, the decoupling increases
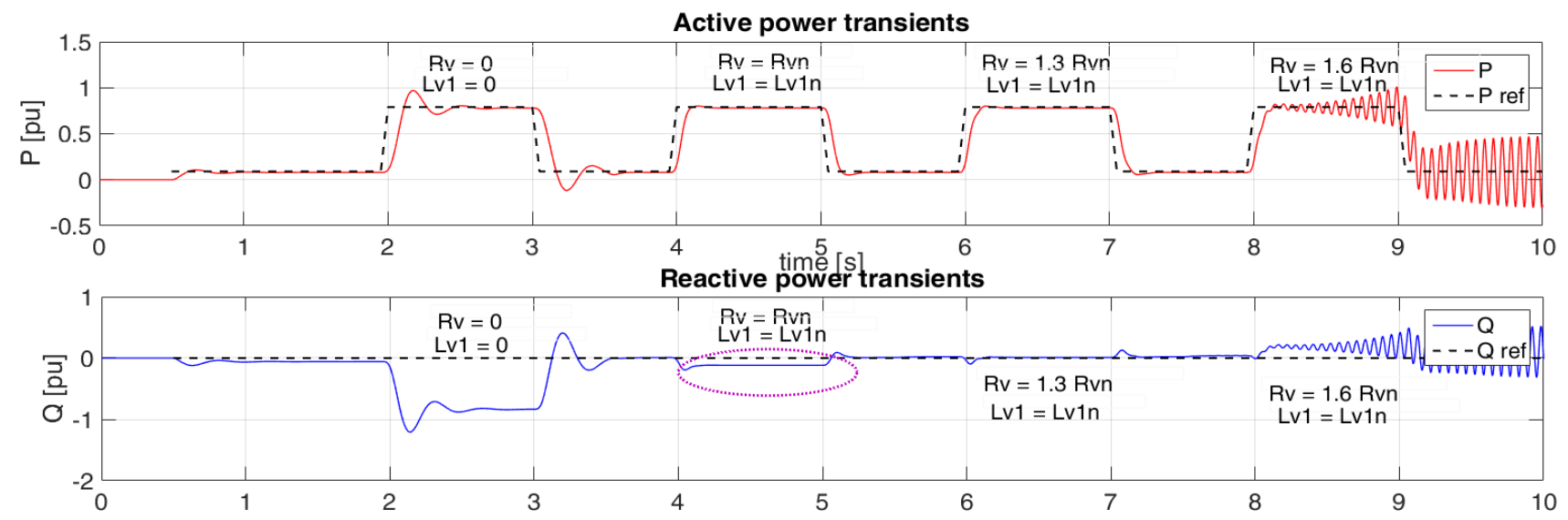

Fig. 7: Transient comparison under step changes of the active power reference with different virtual resistance values. The virtual resistance has been subsequently increased while keeping constant the static virtual reactance. In this simulation the dynamic virtual reactance has not been introduced. In this simulation, the virtual resistance $\mathrm{R}_{\mathrm{V}}$ values are negative while static virtual reactance $j \omega \mathrm{L}_{\mathrm{V} 1}$ are positive, coherently to the design procedure shown before. 

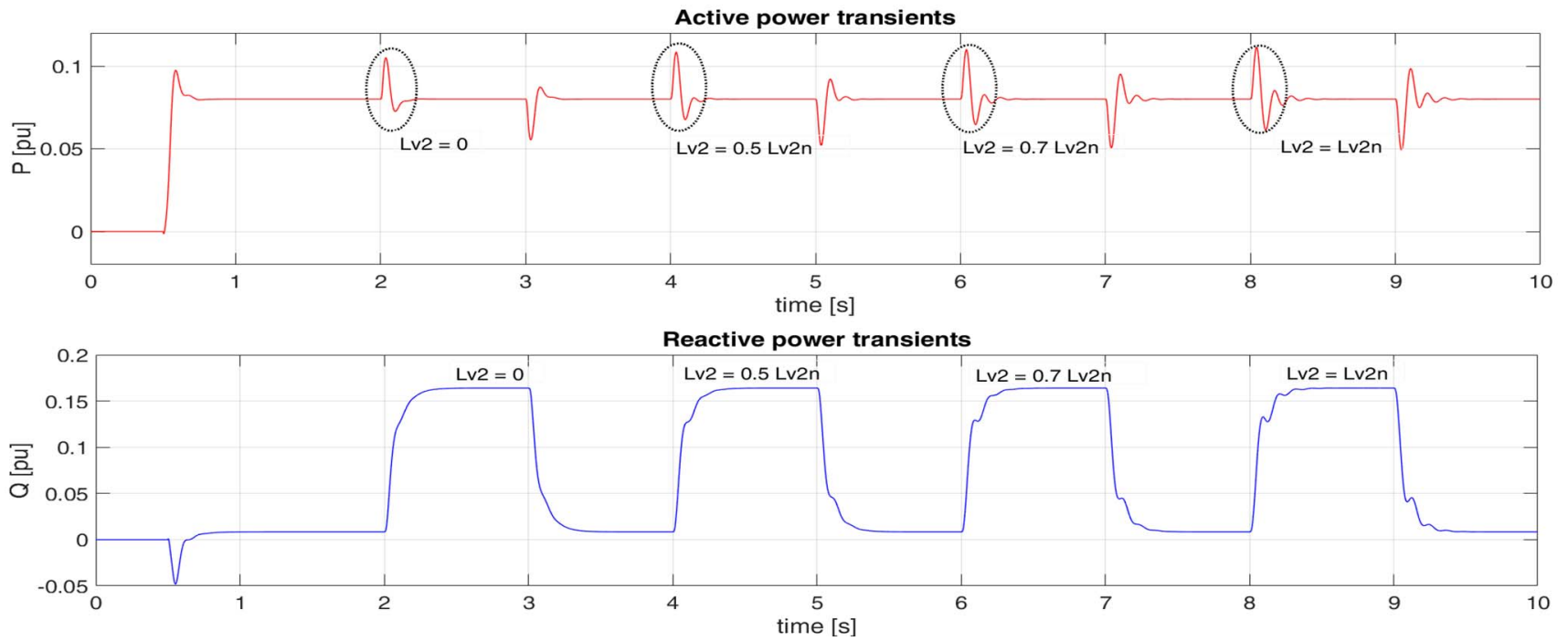

Fig. 8: Transient comparison under step changes of the reative power reference with different values of dynamic virtual reactance. The simulation has been implemented considering $R_{V}=R_{V n}$ and $L_{V 1}=L_{V 1 n}$ and changing the values of $L_{V 2}$, which represents the gain of the dynamic virtual reactance. In this simulation, the virtual resistance $\mathrm{R}_{\mathrm{V}}$ has a negative value, while $\mathrm{L}_{\mathrm{V} 1}$ and $\mathrm{L}_{\mathrm{V} 2}$ are positive, coherently to the design procedure shown before.

even if the phase margin of the voltage control reduces. Even if the decoupling is not complete (Fig. 7 - case 2), the nominal conditions derived in the design process guarantee a certain stability margin for the voltage loop. As the virtual resistance $R_{V}$ becomes more negative, the system tends to become less stable and small oscillations appear in the power transients, which reveal a reduced damping factor for the corresponding eigenvalues. This may lead the system towards instability, as shown in case 4 of Fig. 7.

Fig. 8 shows the results of a simulation which dealing with the effect of the dynamic virtual reactance during system transients induced by step variations of the reactive power reference. First of all it is possible to see that the introduction of dynamic virtual reactance increases the fastness of the control system in following the reference signal. If we consider the initial derivative of the reactive power in the four cases, it is possible to see an increase of $10 \%$ between case 1 and 4 , coherently to the band-pass increase shown in the design guidelines. It is worth to underline that its introduction only acts as a transient term and thus does not produce any significant change in the steady state behaviours for reactive power.

\section{CONCLUSION}

The paper aims at defining a possible design criterion for the virtual impedance loops that keeps into account the closed-loop stability constraints for the converter control system. In particular it has been decided to follow an analytical approach with the goal to identify the effect of each virtual reactance parameter on the controller feedbacks.

The analysis has revealed a strong influence of the virtual resistance on the phase margin of the voltage controller openloop transfer function. A negative value of virtual resistance increases the effectiveness of power decoupling even if it produces a reduction of the voltage loop phase margin. The model has been verified comparing the main findings with the one obtainable from the analysis of the equivalent circuit of the system; moreover the proposed design approach has been tested with a simulation implemented in the Matlab-Simulink environment. The decision to analyse the problem analityically depends on the will to define suitable regulation schemes and simplified approaches to the design issues.

\section{REFERENCES}

[1] A. Micallef, M. Apap, C. Spiteri-Staines and J. M. Guerrero, "Performance comparison for virtual impedance techniques used in droop controlled islanded microgrids," 2016 International Symposium on Power Electronics, Electrical Drives, Automation and Motion (SPEEDAM), Anacapri, 2016, pp. 695-700.

[2] X. Wang, F. Blaabjerg and Z. Chen, "An improved design of virtual output impedance loop for droop-controlled parallel three-phase voltage source inverters," 2012 IEEE Energy Conversion Congress and Exposition (ECCE), Raleigh, NC, 2012, pp. 2466-2473.

[3] A. D. Paquette and D. M. Divan, "Virtual Impedance Current Limiting for Inverters in Microgrids With Synchronous Generators," in IEEE Transactions on Industry Applications, vol. 51, no. 2, pp. 1630-1638, March-April 2015.

[4] G. M. Foglia, L. Frosio, M. F. Iacchetti and R. Perini, "Control loops design in a grid supporting mode inverter connected to a microgrid," 2015 17th European Conference on Power Electronics and Applications (EPE'15 ECCE-Europe), Geneva, 2015, pp. 1-10.

[5] Y. A. R. I. Mohamed and E. F. El-Saadany, "Adaptive Decentralized Droop Controller to Preserve Power Sharing Stability of Paralleled Inverters in Distributed Generation Microgrids," in IEEE Transactions on Power Electronics, vol. 23, no. 6, pp. 2806-2816, Nov. 2008. 